In der Rubrik „Literatur kompakt" werden die wichtigsten Originalarbeiten aus der internationalen Fachliteratur referiert.

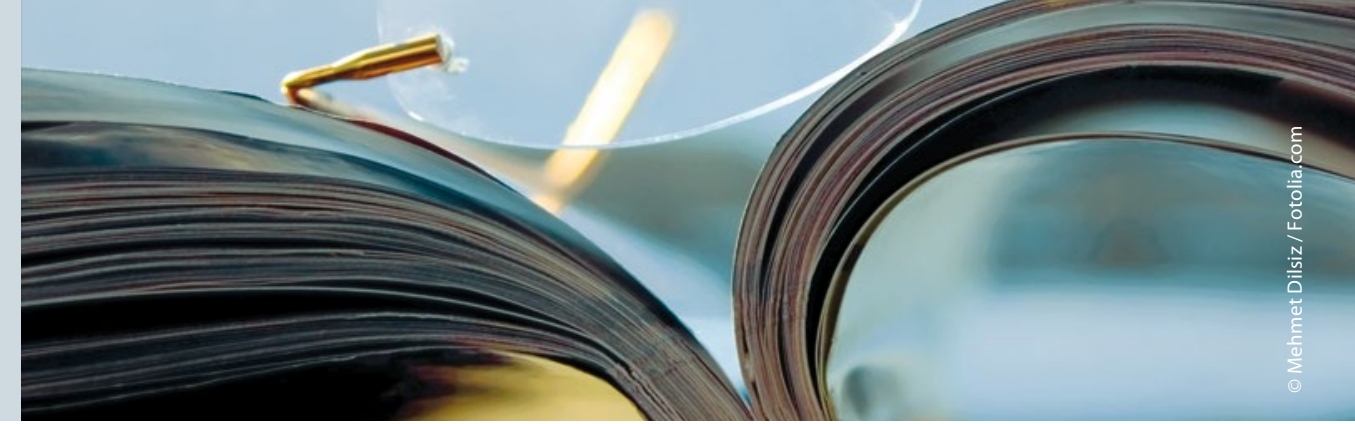

\section{TEP-Standzeiten: hohes Wechselrisiko bei Männern unter 55 Jahren}

\begin{abstract}
Je jünger ein Patient zum Zeitpunkt der Implantation einer Hüft- oder KnieTEP ist, desto größer ist, allein schon wegen der Länge der verbleibenden Lebenszeit, die Wahrscheinlichkeit, dass irgendwann eine Wechseloperation nötig wird; diese Tatsache ist aus mehreren Registerstudien bekannt. Wie jedoch neue Daten aus Großbritannien zeigen, wurde das Risiko einer Revision vor allem bei Patienten unter 60 offenbar deutlich unterschätzt.
\end{abstract}

ee E Bayliss von der Universität Ox- ford und sein Team berufen sich auf Daten von 117.434 Patienten, die im Clinical Practice Research Datalink, einem britischen Register mit insgesamt über sechseinhalb Millionen Patienten der Primärversorgung, erfasst sind. Für die Studie berücksichtigt wurden alle Patienten, die zwischen dem 1. Januar 1991 und dem 10. August 2011 erstmals eine Hüft- oder Knie-Totalendoprothese (TEP) erhalten hatten und zu diesem Zeitpunkt über 50 Jahre alt waren. Diese Altersgrenze wurde gewählt, um die oft besonders komplexen Fälle sehr junger Patienten auszuschließen.

Eine Hüft-TEP hatten im Studienzeitraum 63.158 Patienten erhalten, einKnie-Kunstgelenk 54.276. Das mittlere Alter zum Zeitpunkt des Eingriffs lag bei 69,4 beziehungsweise 70,1 Jahren. $15 \%$ hatten das Kunstgelenk - Hüfte oder Knie - vor dem 60. Lebensjahr erhalten. Ebenfalls $15 \%$ waren 80 oder älter. Zehn Jahre nach der Implantation befanden sich insgesamt noch 95,6\%, nach 20 Jahren noch 85,0 \% der ursprünglichen Prothesen im Körper.

Wie die Autoren berichten, stieg das Lebenszeitrisiko einer Wechseloperation mit abnehmendem Alter der Patien- ten zum Zeitpunkt der Erstimplantation. Am höchsten war die Wahrscheinlichkeit einer Revision bei Männern, die im Alter zwischen 50 und 54 Jahren erstmalig eine Knie-TEP erhalten hatten: Von ihnen benötigten $35 \%$ irgendwann im Laufe ihres Lebens eine neue Prothese. Bei der entsprechenden Gruppe mit Hüft-TEP lag die Revisionsrate bei $29,6 \%$.

Dies stand in deutlichem Kontrast zum Revisionsrisiko bei der Implantation mit 70 Jahren (dem Durchschnittsalter aller Patienten): Hier lag die Rate zwischen $4,4 \%$ und 7,7 \%. Fand die Erstoperation mit 60 statt, war - sowohl für die Hüfte als auch für das Knie - mit einer Revisionsrate von $15 \%$ zu rechnen. Nach Bayliss und Kollegen behält also die größte Gruppe, nämlich Patienten im Alter von 70 und mehr Jahren, ihre Prothese „mit 95\%iger Wahrscheinlichkeit ein Leben lang“. Generell war die Chance, dass das Erstimplantat lebenslang hielt, bei Frauen größer. Bei den Anfang 50-Jährigen lag die Wechselrate im Schnitt um 15 Prozentpunkte niedriger als bei gleichaltrigen Männern.

Bemerkenswert ist für die Autoren auch, wie rasch es in vielen Fällen zum Prothesenversagen kam. Die Austausch- spitze lag über alle Altersgruppen hinweg bei etwa fünf Jahren. Im Mittel dauerte es bei den zum Indexzeitpunkt unter 60-Jährigen 6,56 Jahre bis zur Hüftund 4,55 Jahre bis zur Knierevision; bei Patienten zwischen 70 und 80 waren es 4,08 beziehungsweise 3,57 Jahre. In allen Gruppen waren die Revisionsraten für männliche und jüngere Patienten höher.

Ein Prothesenwechsel innerhalb von fünf Jahren bedeute auch ein erhöhtes Risiko, dass der Eingriff noch ein weiteres Mal wiederholt werden müsse, geben die Autoren zu bedenken. Damit steige für junge Patienten, die vielleicht noch 30 Jahre oder mehr Lebenszeit vor sich haben, die Wahrscheinlichkeit, viele Jahre mehr als erwartet mit einem revidierten Implantat leben zu müssen. In solchen Fällen sei das langfristige Ergebnis besonders schlecht.

Fazit: Für die Autoren ist es eine wichtige Konsequenz ihrer Studienergebnisse, dass Ärzte ihre Patienten vor einer geplanten TEP-Operation gründlicher als bisher über die langfristigen Aussichten informieren sollten. Man müsse die potenziellen Vorteile im Hinblick auf die Lebensqualität gegen die möglichen $\mathrm{Ri}$ siken abwägen. Im Einzelfall sei dabei neben der Revision auch die Gefahr von Operationskomplikationen wie Infektionen und eines eventuell schlechten funktionellen Ergebnisses zu bedenken.

Dr. Elke Oberhofer

Bayliss LE et al. The effect of patient age at intervention on risk of implant revision after total replacement of the hip or knee: a population-based cohort study. Lancet 2017; doi: 10.1016/S0140-6736(17)30059-4 